\title{
Acute Abdomen caused by Perforated Jejunal Diverticulitis
}

\author{
Praveena $\mathrm{R}^{\mathrm{a}}$, Ang $\mathrm{CW}^{\mathrm{a}}$, Hiong Chin Lim ${ }^{\mathrm{a}}$, Wong MPK \\ ${ }^{a}$ Colorectal Unit, Division of General Surgery, University of Malaya Medical Centre, Kuala Lumpur, Malaysia \\ ${ }^{b}$ School of Medical Sciences \& Hospital Universiti Sains Malaysia, Kelantan, Malaysia
}

\begin{abstract}
Keywords
jejunal diverticulitis, perforated jejunum, diverticular abscess

\author{
Corresponding Author \\ Dr. Michael Pak-Kai Wong \\ School of Medical Sciences \& Hospital \\ Universiti Sains Malaysia, 16150 Kubang \\ Kerian, Kelantan, Malaysia \\ Tel No: +6097676774 \\ E-mail: michaelpkwong@usm.my \\ Received: 18 April 2020; Accepted: 31 \\ January 2021 \\ Doi: https://doi.org/10.31436/imjm.v20i3
}

\begin{abstract}
Jejunal diverticulum accounts for only $25 \%$ of small bowel diverticula, and approximately $7 \%$ of these will present as complicated jejunal diverticulitis or perforation. Here, we described a case of jejunal diverticular perforation presented as acute abdominal peritonitis. The computed tomography of the abdomen suggested small bowel perforation and therefore, emergency surgical exploration was performed. Intraoperatively, multiple jejunal diverticulae were found with one forming a localised diverticular abscess. Segmental resection of the diseased segment with primary anastomosis was performed. The patient made an uneventful post-operative recovery. Although complicated jejunal diverticulitis is rare, emergency surgery is often warranted if perforation occurs. Computed tomography is valuable in the diagnosis and preoperative planning especially in an emergency surgical conundrum when there is equivocal clinical peritonitis or perforation.
\end{abstract}

\section{INTRODUCTION}

Small bowel diverticulosis is an uncommon pathology when compared with large bowel diverticulosis. The prevalence of small bowel diverticulosis from autopsy series ranges between $0.3 \%$ to $2.3 \%$, while only less than $4 \%$ of patients with these are symptomatic. ${ }^{1}$ Given its rarity and lacking of consensus guidelines, jejunal diverticulitis remains a challenging pathology to manage. The presence of extra-mural air on computed tomography raises the suspicion of a free perforation that warrants an emergency surgical exploration. However, a small amount of extra-mural air adjacent to the bowel wall may also be secondary to inflammation without macroperforation, which can be managed conservatively without surgery.

Here, we described a case of an obese patient who presented with acute abdomen secondary to complicated jejunal diverticulitis and sealed perforation, and the management of this pathology was discussed.

\section{CASE PRESENTATION}

A 70-year-old man presented to the emergency department with complaints of colicky central abdominal pain for three days associated with fever but no vomiting or altered bowel habits. He had underlying hypertension, diabetes mellitus, dyslipidemia, and ischaemic heart disease that was previously treated with coronary bypass surgery and on lifelong antiplatelet therapy. He also had an asymptomatic reducible paraumbilical hernia.

On physical examination, he was hemodynamically stable but febrile. The central abdomen was tender with voluntary guarding on palpation and the bowel sounds were sluggish.

The blood investigations revealed leucocytosis (total white cell count of $18.3 \times 10^{9} / \mathrm{L}$ ) while the other blood parameters were within the normal range. There was no air under the diaphragm on the erect chest radiograph and the plain supine abdominal radiography revealed no 
features suggestive of dilated bowels. An urgent contrast -enhanced computed tomography (CECT) scan of the abdomen and pelvis was performed, which showed multiple small and large bowel diverticula along with a short segment of jejunum with hypodense irregularities on its wall. There was also the presence of extraluminal air with mesenteric fat stranding, which suggested perforated jejunal diverticulitis.

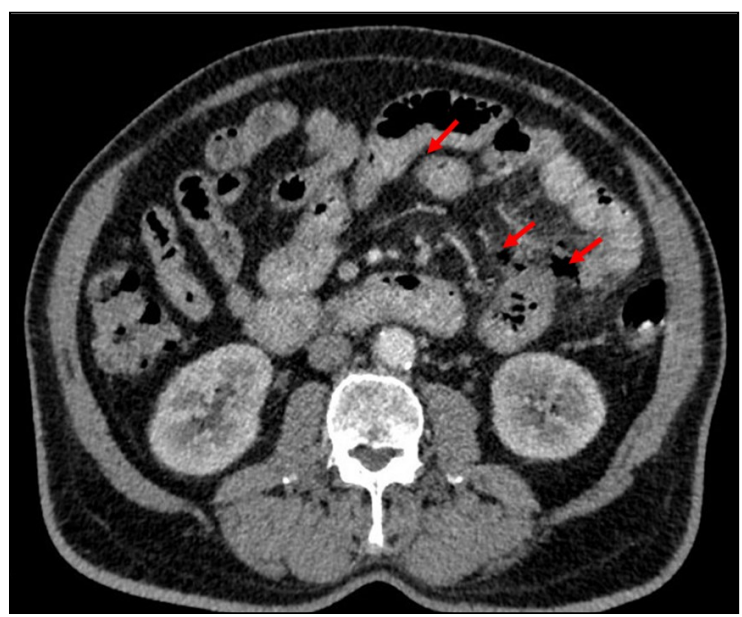

Figure 1: Free extraluminal air demonstrated on contrast -enhanced computed tomography CECT (red arrows)

Given the above findings, an emergency exploratory laparotomy was performed. There were multiple broadbased jejunal diverticula from $10 \mathrm{~cm}$ to $80 \mathrm{~cm}$ of the duodenojejunal (DJ) junction located at the mesenteric border of the jejunum (Figure 2). Among these, a jejunal diverticulum located $80 \mathrm{~cm}$ from the DJ appeared inflamed, thickened and with localised abscess collection (Figure 3). There was no spillage of the bowel content and therefore, suggestive of a sealed perforation. The diseased segment was resected followed by primary anastomosis. The paraumbilical hernia was repaired primarily with tension-free approximation along with the laparotomy wound. He made an uneventful recovery following surgery and was discharged from the hospital after nine days of stay. The subsequent histopathological examination of the resected bowel confirmed the diagnosis of jejunal diverticulitis.

\section{DISCUSSION}

The incidence of small bowel diverticulum is markedly less than the colon that commonly increases with age. The small bowel diverticulum can develop at the duodenum (60-70\%), jejunum (20-25\%), ileum (5-10\%), and multiple sites $(5 \%){ }^{2}$ Although most remain asymptomatic, it is reported that $4-30 \%$ of patients with jejunal diverticulosis may become symptomatic. ${ }^{1,3}$ The clinical presentation of complicated jejunal diverticulosis or perforation is variable, which includes chronic crampy abdominal pain, diverticulitis, abscess formation, bleeding, fistulation, mechanical intestinal obstruction, and perforation. The incidence of jejunal diverticulitis ranges from $0.02 \%$ to $0.42 \%$, with $2.1 \%$ to $7.0 \%$ of patients present with severe sequelae of perforation. 4,5

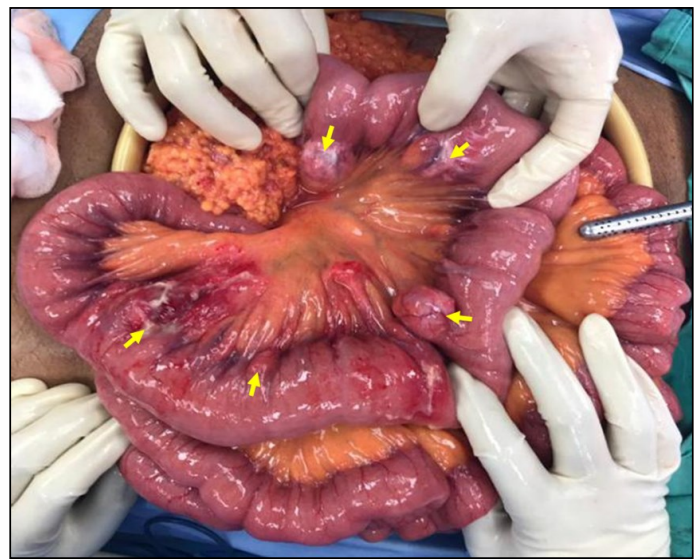

Figure 2: Multiple broad-based diverticulae at the jejunum (yellow arrows)

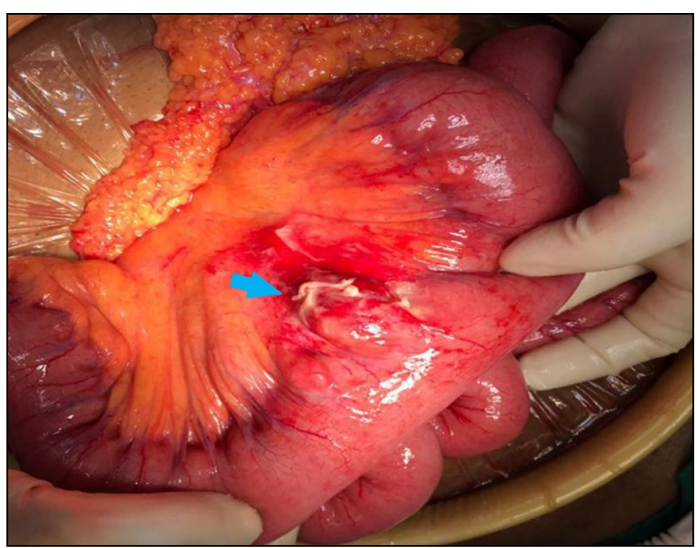

Figure 3: Jejunal diverticulitis at $80 \mathrm{~cm}$ from DJ with localised abscess (blue arrow)

The CECT scan of the abdomen and pelvis remains the gold-standard in the diagnosis of complicated small bowel diverticulitis. ${ }^{5}$ The extraluminal air on CT imaging usually suggests free perforation which therefore, warrants an urgent surgical intervention. In our case, upon laparotomy, we did not observe pneumoperitoneum or macroperforation despite the CECT scan demonstrating free air. Consistent with other reports, these findings were attributed to microperforation with rapidly closed leaking diverticulum or the transmural passage of air through the thin-walled semi-permeable membrane of the diverticulum as observed in any non-Meckelian jejuno- 
ileal diverticulum. ${ }^{6}$ These diverticula are considered false and acquired diverticulum that lacks a true muscular wall and hence, thin-walled and fragile.4,6 Therefore, it is paramount for the clinician to correlate clinical findings with the imaging before embarking on either conservative management with antibiotics or surgical intervention. Nevertheless, a review article has alluded that non-surgical management of complicated jejunal diverticulitis with perforation is rarely effective that it would be better to perform a surgical exploration and limited bowel resection. ${ }^{4}$

Furthermore, the mortality associated with jejunal diverticulitis has been reported to range from $0 \%$ to $5 \%$ but raises exponentially to $40 \%$ in the presence of major perforation and peritonitis. ${ }^{5}$ During surgery, the bowel resection should be limited to the affected complicated segment only (local abscess, peritonitis, or bleeding) if there is an extensive uncomplicated small bowel diverticulosis encountered in order to avoid short bowel syndrome ${ }^{2,4}$, as in our case. When a perforated diverticulum is located adjacent to the duodenojejunal (DJ) junction, a simple closure or diverticulectomy may be performed with a view of balancing between resection and the potential anastomotic morbidity due to the higher pressure system. ${ }^{2,4}$

A systematic review has reported that segmental intestinal resection and primary end-to-end anastomosis with peritoneal lavage has been the most performed technique for the management of complicated small bowel diverticulitis or perforation. ${ }^{2}$ Contrary to the microperforation of colonic diverticulitis that can be managed successfully with laparoscopic-assisted lavage without bowel resection and systemic antibiotics, ${ }^{7}$ this approach has not been described in the literature for treating the microperforation of small bowel diverticulitis. To date, the effective and safest recommended practice for perforated jejunal diverticulitis is segmental resection or diverticulectomy of the affected site.

\section{CONCLUSION}

Jejunal diverticulitis with perforation is very rare. A prompt diagnosis correlating the clinical findings with radiological imaging is key to avoid delay in surgical intervention and subsequent significant morbidity and mortality.

\section{CONFLICT OF INTEREST}

We declare no conflict of interest

\section{ACKNOWLEDGEMENT}

None

\section{REFERENCES}

1. Singh O, Gupta SS, Shukla S, Mathur RK, Shukla S. Jejunal diverticulae: Reports of two cases with review of literature. Indian J Surg 2009; 71: 238244.

2. de Sumone B, Akberucu K, Ansakonu K, Sartekku M, Coccokunu F, Catena F. Not all diverticulites are colonic: Small bowel diverticulitis - A systematic review. Minerva Chir 2019; 74: 137145.

3. Butler JS, Collins CG, McEntee GP. Perforated jejunal diverticula: A case report. J Med Case Rep 2010; 4: 2-4.

4. Harbi H, Kardoun N, Fendri S, Dammak N, Toumi N, Guirat A et al. Jejunal diverticulitis. Review and treatment algorithm. Press Medicale 2017; 46: 1139-1143.

5. Kwak JY, Park EH, Park CS, Kim JH, Han MS, Kwak JH. Uncomplicated jejunal diverticulosis with pneumoperitoneum. Ann Surg Treat Res 2016; 90: 346-349.

6 Syllaios A, Koutras A, Zotos PA, Triantafyllou E, Bourganos N, Koura S et al. Jejunal Diverticulitis Mimicking Small Bowel Perforation: Case Report and Review of the Literature. Chirurgia (Bucur) 2018; 113: 576.

7 Myers E, Hurley M, O’Sullivan GC, Kavanagh D, Wilson I, Winter DC. Laparoscopic peritoneal lavage for generalized peritonitis due to perforated diverticulitis. Br J Surg 2008; 95: 97-101. 